\title{
Imaging of Anaplastic Thyroid Carcinoma
}

\author{
(D) S. Ahmed, (D) M.P. Ghazarian, (D) M.E. Cabanillas, (D) M.E. Zafereo, (D) M.D. Williams, (DT. Vu, (DD.F. Schomer, and (D).M. Debnam
}

\begin{abstract}
SUMMARY: Anaplastic thyroid carcinoma is fatal if unresectable. However, improved survival has been reported after gross total resection and multimodality therapy. In this report, we describe the contrast-enhanced high-resolution CT characteristics of anaplastic thyroid carcinoma in 57 patients. Anaplastic thyroid carcinoma presented as a large neck mass with necrosis in $82 \%$ of cases. The tumors demonstrated common extrathyroidal extension (91\%). Sixty-two percent of tumors demonstrated calcification. Visceral space invasion involved the esophagus (62\%), trachea (57\%), and larynx (29\%). Carotid artery encasement was present in $42 \%$, and $43 \%$ involved the internal jugular vein. Sixty-three percent had lateral compartment lymphadenopathy; $58 \%$ of these nodes were necrotic, and $11 \%$ were cystic. No metastatic nodes had calcification. Central compartment lymphadenopathy was seen in $56 \%$ of cases, and lateral retropharyngeal lymphadenopathy was detected in $12 \%$. Knowledge of these imaging features aids in guiding the approach to the initial tissue diagnosis with either fine-needle aspiration or core biopsy, assessing the feasibility of surgical resection, and determining prognosis.
\end{abstract}

ABBREVIATIONS: ATC = anaplastic thyroid carcinoma; CECT = contrast-enhanced CT; DTC = differentiated thyroid cancer

A naplastic thyroid carcinoma (ATC) accounts for $1 \%-2 \%$ of all thyroid malignancies, but results in over $50 \%$ of deaths from thyroid cancer. ${ }^{1-3}$ Despite modern therapies, it remains a highly aggressive disease with one of the worst prognoses among all malignancies. Historically, mean survival was approximately only 5 months, ${ }^{4}$ and 1 -year survival was $20 \%$, with 2 -year survival at approximately $10 \% .^{5}$ With recent advances in multidisciplinary care, median survivals have improved, though most patients do not achieve ultimate cure of their disease. ${ }^{6}$ Women are affected slightly more than men at a ratio of $1.5: 1$, with a peak incidence in the sixth and seventh decades of life. ${ }^{7,8}$ ATC is known to arise through 2 mechanisms: de novo or from dedifferentiation of well-differentiated thyroid cancer. ${ }^{9,10}$

ATC is characterized by local invasiveness and extensive ne-

Received August 4, 2017; accepted after revision October 22.

From the Departments of Diagnostic Radiology (S.A., T.V., D.F.S., J.M.D.), Endocrine Neoplasia (M.E.C.), Head and Neck Surgery (M.E.Z.), and Pathology (M.D.W.), University of Texas MD Anderson Cancer Center, Houston, Texas; and Department of Diagnostic and Interventional Imaging (M.P.G.), University of Texas Houston Medical School, Houston, Texas.

This work was supported by The University of Texas MD Anderson Cancer Center's Cancer Center Support Grant CA16672.

Paper previously presented at: Annual Meeting of the American Roentgen Ray Society, April 17-22, 2016; Los Angeles, California, and Annual Meeting of the American Society of Neuroradiology, May 23-26, 2016; Washington, DC.

Please address correspondence to Salmaan Ahmed, MD, Department of Diagnostic Radiology, 1400 Pressler St, Unit 1482, Houston, TX 77030; e-mail: salmaan.ahmed@mdanderson.org

http://dx.doi.org/10.3174/ajnr.A5487 crosis. ${ }^{11}$ The typical presentation is that of a rapidly enlarging neck mass, often in older patients, prompting contrast-enhanced CT (CECT) evaluation. ${ }^{12}$ Doubling time for ATC tumor volume can be as low as 1 week. ${ }^{1,13}$ Symptoms of mass effect upon the trachea and larynx are usually seen at initial presentation. Direct invasion of adjacent structures, including muscle, trachea, larynx, esophagus, and recurrent laryngeal nerve, is common. In addition, nodal metastases are seen in up to $40 \%$ of patients at diagnosis. ${ }^{14}$ Distant metastases are present in $43 \%$ of patients upon presentation, with the most common locations being the lung (78\%), adrenals (24\%), liver (20\%), and brain (18\%). ${ }^{14}$ Of identifiable causes, death is most commonly the result of advanced pulmonary metastatic disease (35\%), followed by airway compromise $(16 \%)$, tumor-related hemorrhage (14\%), and cardiac failure $(11 \%) .^{8}$

In distinction to differentiated thyroid cancer (DTC) and medullary thyroid cancer, all cases of ATC are staged by the American Joint Committee on Cancer TNM system as stage IV disease because of its aggressive nature and poor prognosis. ${ }^{4,8}$ Stage IVA denotes primary tumor without extrathyroidal extension and is considered resectable disease. Stage IVB denotes tumor extension beyond the thyroid without distant metastasis and may or may not be resectable depending on the extent of local invasion. Stage IVC indicates distant metastatic disease. Given improved survival with early multimodality treatment ${ }^{6}$ and increased use of head and neck imaging, it is important for radiologists to be familiar with the imaging features of ATC to avoid delays in diagnosis. In 
addition, defining the extent of disease, including local invasion and metastatic adenopathy, is critical for appropriate management of the patient.

The imaging literature on ATC is limited, with most reports from before the advent of multidetector CT. ${ }^{15,16}$ To our knowledge, the single article in the radiology literature describing the imaging findings of ATC with multidetector CT included only 9 cases and did not address the frequency of organ invasion, vascular encasement, and cervical lymphadenopathy. ${ }^{17}$ Therefore, the purpose of our study was to accurately describe the CECT features of ATC at presentation in a large number of patients presenting to a tertiary cancer referral center.

\section{MATERIALS AND METHODS}

Under an institutional review board-approved protocol, the institutional data base was queried for patients with the diagnosis of anaplastic thyroid cancer between June 2004 and February 2017. Patients without CECT imaging before any therapy for ATC or those with suboptimal image quality were excluded. Fifty-seven patients with biopsy-proved ATC and CECT before therapy were included in this retrospective review. Patients underwent imaging on a 4-16-detector LightSpeed CT scanner (GE Healthcare, Milwaukee, Wisconsin), with axial $1.25-3 \mathrm{~mm}$ collimation at 120 $\mathrm{kVp}$ and $160-400 \mathrm{~mA}$, after injection of $125 \mathrm{~mL}$ of Omnipaque 350 (GE Healthcare) at $3 \mathrm{~mL} / \mathrm{s}$ with a 90 -second delay.
Two neuroradiologists (S.A. and either J.M.D. or T.V.), each with a Certificate of Added Qualification and subspecialty interest in head and neck imaging, reviewed all CECT images by consensus. Images were evaluated for the presence of the following: 1) extent of tumor involvement within the thyroid; 2) size of tumor (anteroposterior and transverse dimensions in the axial plane); 3 ) margins; 4) extrathyroidal extension; 5) tumor morphology; 6) presence of calcifications; 7) esophageal invasion; 8) tracheal invasion; 9) laryngeal invasion; 10) carotid encasement; 11) internal jugular vein involvement; and 12) intrathoracic extension. The presence of central compartment, lateral compartment, and lateral retropharyngeal lymphadenopathy was documented. Lateral and central compartment lymphadenopathy were assessed for calcification, necrosis, and cystic change.

\section{RESULTS}

Of the 207 patients with the diagnosis of ATC, 60 patients had pretherapy imaging. Three of the 60 patients were excluded for poor image quality. Of the 57 patients reviewed in our study, 30 patients were men and 27 patients were women. The patient ages ranged between 21 and 82 years (mean, 64 years; median, 65 years). The mean size of the primary tumor was $5.1 \times 4.5 \mathrm{~cm}$ with a median size of $5.0 \times 4.5 \mathrm{~cm}$ (anteroposterior $\times$ transverse). Figure 1 shows the baseline imaging characteristics of the primary tumor in our patient population, and representative images from 1 of our study patients are shown in Fig 2. The tumor involved 1 lobe of the thyroid in 26 patients $(46 \%)$, and both lobes were involved in 31 patients (54\%). Tumor margins were ill-defined in 47 patients $(82 \%)$ and well-defined in 10 patients $(18 \%)$.

Extrathyroidal extension of the tumor was present in 52 of 57 patients (91\%). The tumor morphology was mixed solid and necrotic in 47 patients (82\%), with the remainder being solid. No lesions were purely cystic. Calcification was present in $62 \%$ of cases $(35 / 57)$. Of those tumors with calcification, coarse calcification was the most com-

FIG 1. Anaplastic thyroid carcinoma characteristics.
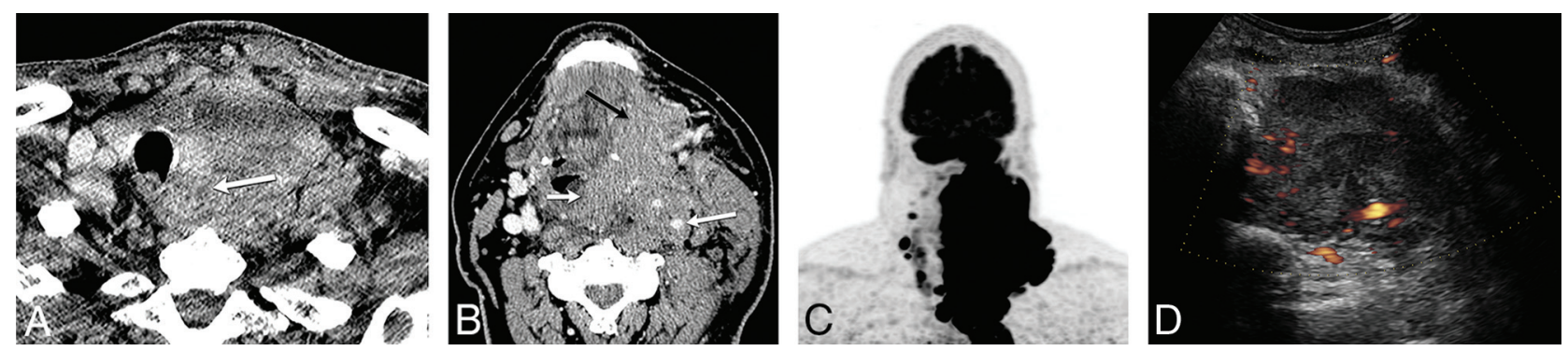

FIG 2. A 60-year-old man with history of neck pain for 1 month and neck CT demonstrating a large left neck mass biopsied as anaplastic thyroid cancer, stage IVC. A, CECT demonstrates a heterogeneously enhancing solid tumor involving the left thyroid lobe and isthmus, with extension to the tracheoesophageal groove and esophageal invasion (arrow). B. Tumor encases the internal carotid artery (large white arrow), and there is direct extension to the prevertebral space, supraglottis (small white arrow), oropharynx, and floor of mouth (black arrow). C, FDG-PET shows intense radiotracer uptake within the left neck tumor with extension to the mediastinum, and there, nodal metastasis to the right neck. $D$, Pretreatment sonography with Doppler demonstrates a hypoechoic, solid, diffusely infiltrative mass involving the left lobe with increased vascular flow. 


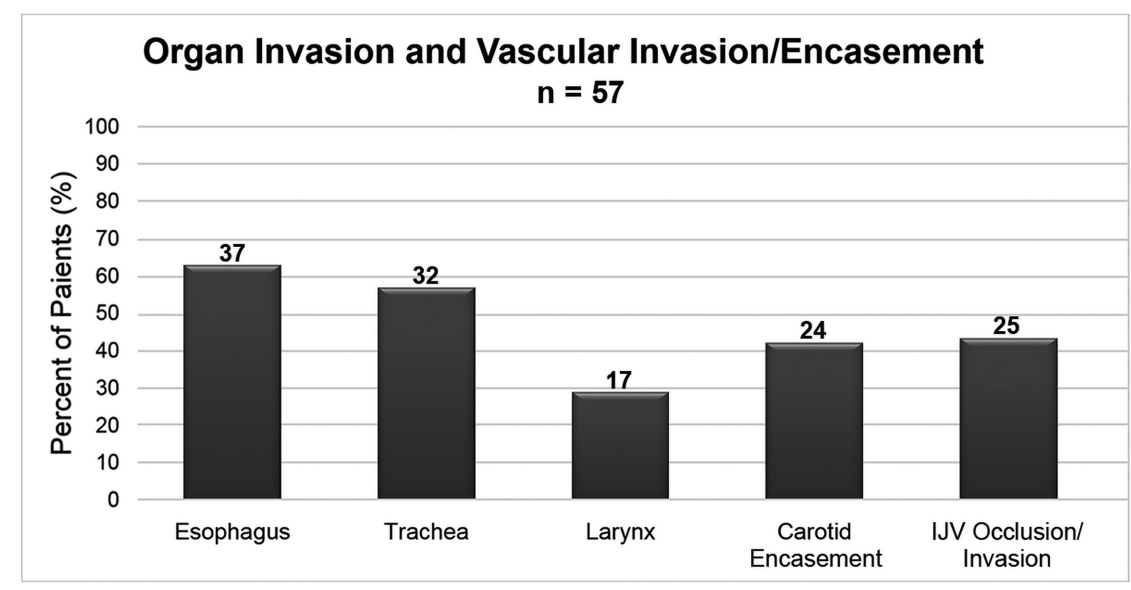

FIG 3. Anaplastic thyroid carcinoma propensity for organ invasion and vascular involvement.
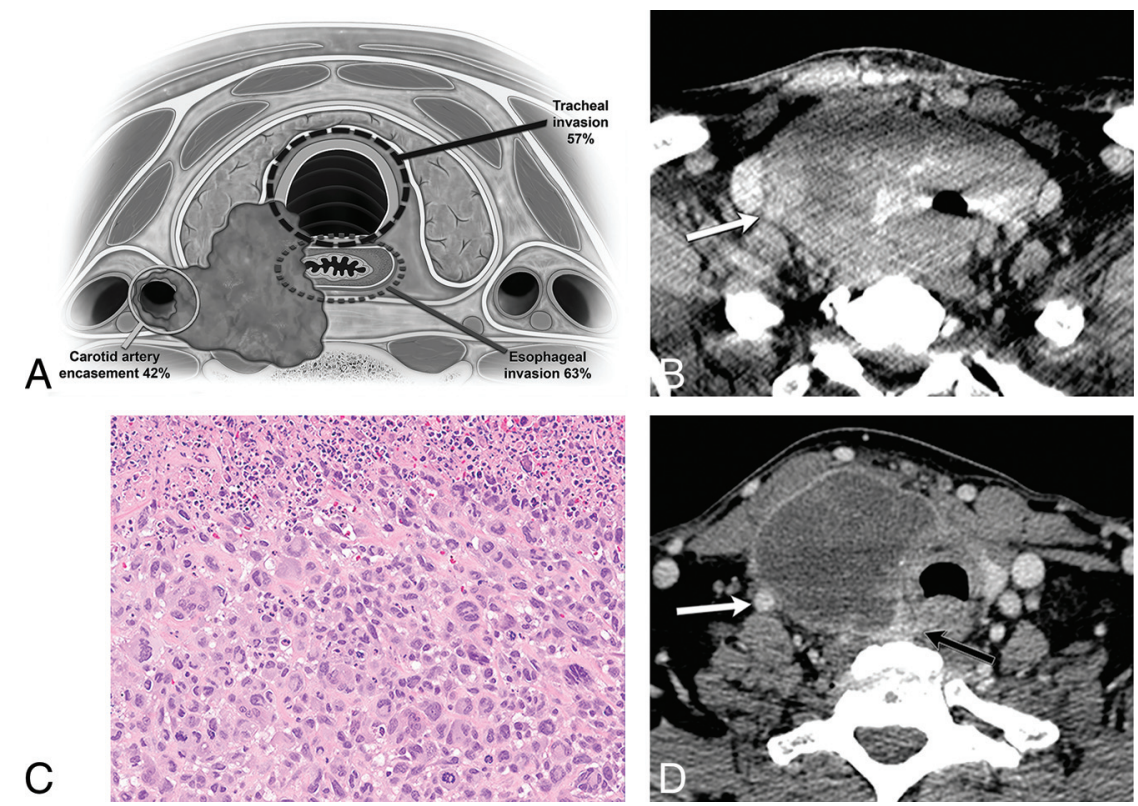

FIG 4. A 60-year-old man with enlarging right neck mass and hoarseness for 2 months with core biopsy demonstrating ATC, stage IVC. A, Graphic demonstrating the critical areas of evaluation to characterize the local invasiveness of an aggressive thyroid mass. $B$, Solid, heterogeneously enhancing right lobe tumor invades the trachea and esophagus, with leftward displacement of the midline structures, and encasement of the right ICA (arrow). C, Histologic features show enlarged, pleomorphic giant cells with hyperchromatic nuclei growing in sheets consistent with ATC. Necrosis with inflammation is common in these tumors as seen in the upper portion of this picture (hematoxylin-eosin tissue section at $200 \times$ magnification). D, After systemic therapy, the tumor mass has significantly decreased in size, with increased central necrosis, and improved overall local invasiveness. The right ICA is no longer encased (white arrow), and the tumor is now separable from the esophagus (black arrow).

mon (20/57), followed by punctate/stippled calcification (19/57) and 5 cases of eggshell calcification (some tumors had more than 1 type of calcification).

Figure 3 shows the frequency of direct organ invasion and vascular involvement by ATC. The organ with the highest propensity for invasion was the esophagus (63\%), followed by the trachea (57\%) and the larynx (29\%). Vascular involvement by the primary tumor was also commonly present, with carotid artery encasement in $42 \%$ of cases and internal jugular vein involvement in $43 \%$. Direct intrathoracic extension of ATC was present in almost half the cases (24/57).
Lateral compartment lymphadenopathy was seen in $63 \%$ of our cases, and these metastatic nodes were frequently necrotic $(58 \%)$, but none contained calcification. Cystic nodes were uncommon (11\%). Central compartment lymphadenopathy was seen in $56 \%$ of cases; $59 \%$ of these nodes were necrotic, and only 3 nodes had calcification (9\%), with 2 being cystic (6\%). Lateral retropharyngeal adenopathy was seen in 7 patients $(12 \%)$.

\section{DISCUSSION}

Although ATC carries a dismal prognosis, improved survival can be achieved in select cases with a gross total resection in combination with chemotherapy/targeted therapy and radiation therapy. Therefore, precise tumor localization is imperative in determining and assessing treatment options. The primary factors that limit the possibility of meaningful surgery for a patient with ATC are carotid artery encasement $(42 \%$ in our study), significant laryngeal and tracheal (57\% and 29\% in our study) or esophageal involvement (63\% in our study), significant mediastinal extension, or significant regional or distant metastases. Although laryngotracheal and esophageal resections may be considered for less biologically aggressive tumors, these more complex surgeries and reconstructions are rarely attempted for patients with ATC because of the morbidity of the surgery in the setting of an overall very poor prognosis. Carotid encasement, which was present in $42 \%$ of our cases, would be considered inoperable. Similarly, reporting vascular involvement is also critical for the oncologist to counsel patients because this radiographic finding indicates the potential for local hemorrhage and death. Figure 4 demonstrates the typical imaging appearance of ATC at presentation in a patient who was initially deemed inoperable. After positive response to chemoradiation therapy, the carotid encasement and deep organ invasion were significantly improved. In a retrospective review of 121 patients with ATC, age $<60$ years, tumor size $<7 \mathrm{~cm}$, and less extensive disease at presentation were independently associated with decreased disease-related mortality. ${ }^{18}$ Increased survival is associated with surgical resection when feasible, younger age, smaller tumor size, higher dose of radiation therapy, absence of distant metastases at presentation, coexistence of papillary thyroid cancer, and management by using multimodal therapy. ${ }^{5,19}$ A retro- 

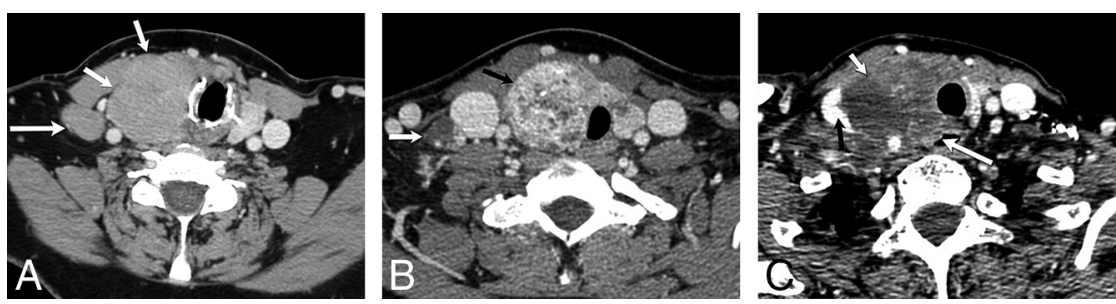

FIG 5. A, Lymphoma: CECT demonstrates a homogeneously enhancing mass diffusely enlarging the right thyroid lobe (small arrows) without calcification or necrosis. There is displacement of midline structures without organ invasion. Enlarged right level IV node (large arrow) enhances homogeneously without calcification, necrosis, or cystic change. B, Papillary thyroid carcinoma: CECT demonstrates a heterogeneously enhancing right thyroid lobe mass (black arrow) with foci of calcification and without necrosis, displacing the midline structures toward the left without organ invasion. Metastatic right level IV node (white arrow) demonstrates calcification and cystic change. C, Anaplastic thyroid carcinoma: CECT demonstrates a necrotic right thyroid lobe mass (small white arrow) directly invading the esophagus (large white arrow) and internal jugular vein (black arrow), and there is encasement of the common carotid artery.
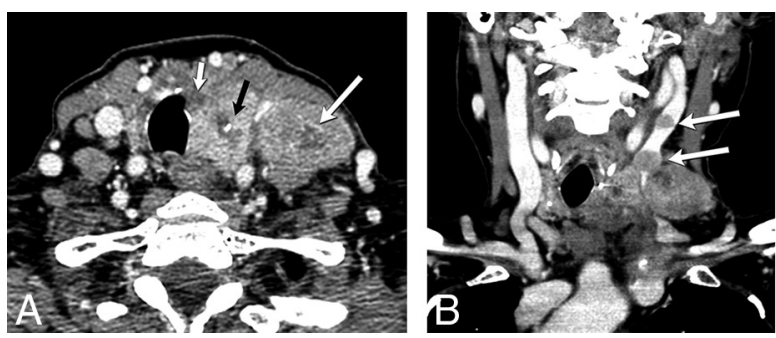

FIG 6. A 64-year-old woman with fine-needle aspiration of left level IV node demonstrating anaplastic thyroid carcinoma, stage IVB. Patient subsequently underwent thyroidectomy, and surgical pathology demonstrated anaplastic thyroid carcinoma arising within papillary thyroid carcinoma. A, Preoperative CECT demonstrates heterogeneously enhancing nodule within the left thyroid lobe with calcification likely corresponding to the pre-existing papillary thyroid carcinoma (black arrow). Necrotic tumor within the anteromedial aspect of the left lobe (small white arrow) may be invading the trachea and likely corresponds to the anaplastic component seen at final pathology. Metastatic left level IV node demonstrates a necrotic focus (large white arrow). B, There is tumor thrombus within the internal jugular vein (arrows). C, Patient underwent total thyroidectomy and bilateral neck dissection, including resection of the left internal jugular vein.

spective review by Mohebati et $\mathrm{al}^{9}$ showed a 1 -year survival of $54 \%$ with gross total resection of the primary tumor versus $28 \%$ with gross residual and $8 \%$ with no surgery.

When encountering a large thyroid mass, the primary concern remains DTC, which represents $>90 \%$ of all thyroid cancers. The differential diagnosis also includes ATC and thyroid lymphoma (particularly when the presentation is a rapidly growing neck mass), and other primary thyroid malignancies (poorly differentiated thyroid carcinoma or medullary thyroid cancer), and less commonly metastasis to the thyroid. Sonography is the preferred initial technique for evaluating a neck mass of thyroid origin; however, patients with a rapidly enlarging neck mass and symptoms that might include hoarseness and dysphagia (as seen in ATC) frequently undergo initial evaluation with CECT. Sonography, however, is useful in guiding percutaneous biopsy for rapid diagnosis. The sonographic appearance of ATC is nonspecific and typically reveals a large, heterogeneous, hypoechoic, solid mass with vascularity. ${ }^{11}$ Accurate diagnosis of ATC can be achieved with fine-needle aspiration in $84 \%$ of cases. ${ }^{20}$ However, at our institution, core biopsy is preferred because of the extensive necrosis typically seen with ATC and also to facilitate adequate tissue for molecular testing. ${ }^{4}$ Molecular testing, though not recommended in professional guidelines at this time, has gained critical

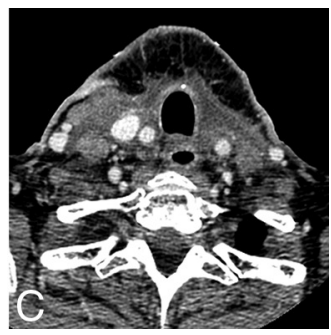

importance in ATC recently. This is because of the recent finding of a basket trial with a BRAF/MEK inhibitor combination (dabrafenib/trametinib) with very high initial response rate in BRAFmutated ATC. A high index of suspicion for ATC when encountering a rapidly enlarging thyroid mass in an older person, and proceeding with a core biopsy aimed at solid non-necrotic areas, improves diagnostic material to triage patients earlier for therapy. At our institution, core biopsy of suspected ATC or thyroid lymphoma is performed under ultrasound guidance by using a 19gauge introducer needle and a 20-gauge biopsy gun.

In addition to high-quality CECT of the neck, the initial staging work-up for ATC would also include whole body imaging with PET-CT to exclude distant metastatic disease, brain MR imaging, and chest CT. ${ }^{2,11}$ Figure 1 demonstrates the typical CT imaging features of ATC at presentation, including a large necrotic mass involving both lobes of the thyroid with poorly defined margins, extrathyroidal extension, and local invasion. These imaging features are uncommon in DTC and raise the concern for a higher-grade thyroid carcinoma (poorly differentiated or anaplastic). Figure 5 illustrates the differentiating CT features of papillary thyroid cancer, lymphoma, and ATC in patients with thyroid masses of similar size at presentation. DTC is most commonly seen on CECT as a solid, homogeneous, hypoattenuating nodule. Calcifications may be seen in both papillary thyroid cancer (32\%) and ATC (62\% in our cohort). ${ }^{21}$ Thyroid lymphoma typically presents with a rapidly enlarging anterior neck mass and symptoms of mass effect, similar to ATC. Differentiation of lymphoma from ATC is important for both prognosis and treatment implications, and when this rare entity is suspected, flow cytometry can be performed on a fresh fine-needle aspiration sample for diagnosis. Ishikawa et $\mathrm{al}^{15}$ described 3 features on CT that differentiated primary thyroid lymphoma from ATC with statistical significance. These included the presence or absence of calcification and necrosis and heterogeneous or homogeneous attenuation. ${ }^{15,21}$ ATC contained calcification and necrosis and had heterogeneous attenuation. Thyroid lymphoma, in contrast, was homogeneous without calcification or necrosis. ${ }^{15}$ Our findings in ATC (Figs 2 and 3) align with those described in ATC by Kim. ${ }^{21}$ Core biopsy is helpful in the characterization of both ATC and lymphoma.

Metastatic lymphadenopathy from ATC is frequently necrotic, and nodal necrosis was present in $58 \%$ of cases in our study. Lymphadenopathy from DTC can be homogeneously enhancing and solid or cystic. Cystic nodes were uncommon in our 
study of ATC and were likely related to metastasis from coexisting DTC. DTC lymph nodes are rarely necrotic. ${ }^{21}$ Thus, it is important for biopsy purposes to target a viable tumor focus, but because ATC and papillary thyroid cancer may coexist ${ }^{22}$ (Fig 6), the lesions with higher-grade imaging features would be preferentially selected for biopsy. If a lymph node is biopsied and initial fine-needle aspiration triage indicates DTC in a patient with CECT imaging of concern for ATC, another biopsy of the thyroid mass would be indicated. Alternatively, if lymph node biopsy returns ATC, no additional sampling of the thyroid mass would be necessary.

\section{CONCLUSIONS}

The presence of a rapidly enlarging necrotic thyroid mass should raise concern for ATC, particularly when there is local organ invasion and necrotic cervical lymphadenopathy. Awareness of these imaging features of ATC allows for radiographic distinctions to favor ATC over a well-differentiated thyroid carcinoma or thyroid lymphoma and potentially expedite diagnosis. The frequent local and regional imaging findings, in particular the tumor extent and association with critical structures in the neck, emphasize the role for high-quality CECT imaging. Knowledge of these imaging features will aid in guiding the approach to initial tissue diagnosis with either fine-needle aspiration or core biopsy, assessing if surgical resection is feasible, and determining prognosis.

Disclosures: Maria E. Cabanillas—UNRELATED: Consultancy: Eisai, LOXO, Blueprint; Grants/Grants Pending: Eisai, Kura, Genentech*. *Money paid to the institution.

\section{REFERENCES}

1. Are C, Shaha AR. Anaplastic thyroid carcinoma: biology, pathogenesis, prognostic factors, and treatment approaches. Ann Surg Oncol 2006;13:453-64 CrossRef Medline

2. Bogsrud TV, Karantanis D, Nathan MA, et al. 18F-FDG PET in the management of patients with anaplastic thyroid carcinoma. Thyroid 2008;18:713-19 CrossRef Medline

3. Ragazzi M, Ciarrocchi A, Sancisi V, et al. Update on anaplastic thyroid carcinoma: morphological, molecular, and genetic features of the most aggressive thyroid cancer. Int J Endocrinol 2014;2014: 790834 CrossRef Medline

4. Smallridge RC, Ain KB, Asa SL, et al. American Thyroid Association guidelines for management of patients with anaplastic thyroid cancer. Thyroid 2012;22:1104-39 CrossRef Medline

5. Smallridge RC, Copland JA. Anaplastic thyroid carcinoma: patho- genesis and emerging therapies. Clin Oncol (R Coll Radiol) 2010;22: 486-97 CrossRef Medline

6. Rao SN, Zafereo M, Dadu R, et al. Patterns of treatment failure in anaplastic thyroid carcinoma. Thyroid 2017;27:672-81 CrossRef Medline

7. Aschebrook-Kilfoy B, Ward MH, Sabra MM, et al. Thyroid cancer incidence patterns in the United States by histologic type, 19922006. Thyroid 2011;21:125-34 CrossRef Medline

8. Neff RL, Farrar WB, Kloos RT, et al. Anaplastic thyroid cancer. Endocrinol Metab Clin North Am 2008;37:525-38, xi CrossRef Medline

9. Mohebati A, Dilorenzo M, Palmer F, et al. Anaplastic thyroid carcinoma: a 25-year single-institution experience. Ann Surg Oncol 2014;21:1665-70 CrossRef Medline

10. Haddad RI, Lydiatt WM, Ball DW, et al. Anaplastic thyroid carcinoma, version 2.2015. J Natl Compr Canc Netw 2015;13:1140-50 CrossRef Medline

11. Hahn SY, Shin JH. Description and comparison of the sonographic characteristics of poorly differentiated thyroid carcinoma and anaplastic thyroid carcinoma. J Ultrasound Med 2016;35:1873-79 CrossRef Medline

12. Cabanillas ME, McFadden DG, Durante C. Thyroid cancer. Lancet 2016;388:2783-95 CrossRef Medline

13. Ain KB. Anaplastic thyroid carcinoma: a therapeutic challenge. Semin Surg Oncol 1999;16:64-69 CrossRef Medline

14. Kelil T, Keraliya AR, Howard SA, et al. Current concepts in the molecular genetics and management of thyroid cancer: an update for radiologists. Radiographics 2016;36:1478-93 CrossRef Medline

15. Ishikawa $\mathrm{H}$, Tamaki $\mathrm{Y}$, Takahashi $\mathrm{M}$, et al. Comparison of primary thyroid lymphoma with anaplastic thyroid carcinoma on computed tomographic imaging. Radiat Med 2002;20:9-15 Medline

16. Takashima S, Morimoto S, Ikezoe J, et al. CT evaluation of anaplastic thyroid carcinoma. AJR Am J Roentgenol 1990;154:1079-85 CrossRef Medline

17. Lee JW, Yoon DY, Choi CS, et al. Anaplastic thyroid carcinoma: computed tomographic differentiation from other thyroid masses. Acta Radiol 2008;49:321-27 CrossRef Medline

18. Kim TY, Kim KW, Jung TS, et al. Prognostic factors for Korean patients with anaplastic thyroid carcinoma. Head Neck 2007;29: 765-72 CrossRef Medline

19. Smallridge RC, Marlow LA, Copland JA. Anaplastic thyroid cancer: molecular pathogenesis and emerging therapies. Endocr Relat Cancer 2009;16:17-44 CrossRef Medline

20. Us-Krasovec M, Golouh R, Auersperg M, et al. Anaplastic thyroid carcinoma in fine needle aspirates. Acta Cytol 1996;40:953-58 CrossRef Medline

21. Kim DW. Computed tomography features of papillary thyroid carcinomas. J Comput Assist Tomogr 2014;38:936-40 CrossRef Medline

22. Molinaro E, Romei C, Biagini A, et al. Anaplastic thyroid carcinoma: from clinicopathology to genetics and advanced therapies. Nat Rev Endocrinol 2017;13:644-60 CrossRef Medline 